\title{
The Misinterpretation of COVID-19 metrics
}

\author{
Gilbert Berdine MD
}

The two most common metrics for the COVID-19 pandemic are deaths and cases. A careful examination of raw data from the Centers for Disease Control (CDC) illustrate difficulties in using these metrics. A recent CDC report gives raw U.S. data for deaths attributed to COVID-19, total deaths, and population stratified by age group as of September 9, 2020. ${ }^{1}$

Figure 1 illustrates the raw data for U.S. deaths attributed to COVID-19 stratified by age group. This figure shows that the raw number of deaths increase with age group. The population is not evenly divided by each age group. Total deaths are not evenly divided by age group. Two immediate questions suggested by this Figure are: what percentage of deaths in each age group are attributable to COVID-19; and what is the mortality rate (deaths per million population) attributable to COVID-19 for each age group?

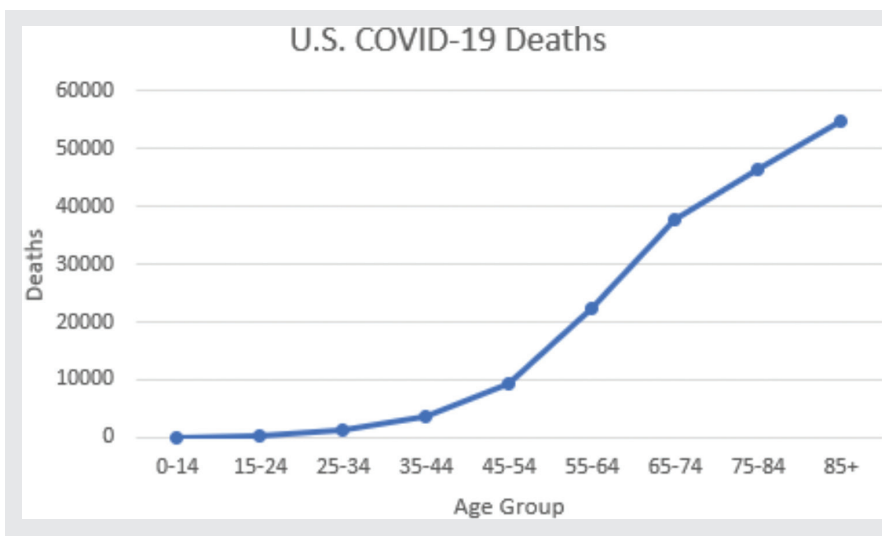

Figure 1. U.S. deaths from COVID-19 by age group. Data are from CDC. ${ }^{1}$

Corresponding author: Gilbert Berdine Contact Information: Gilbert.Berdine@ttuhsc.edu DOI: 10.12746/swrccc.v8i36.755

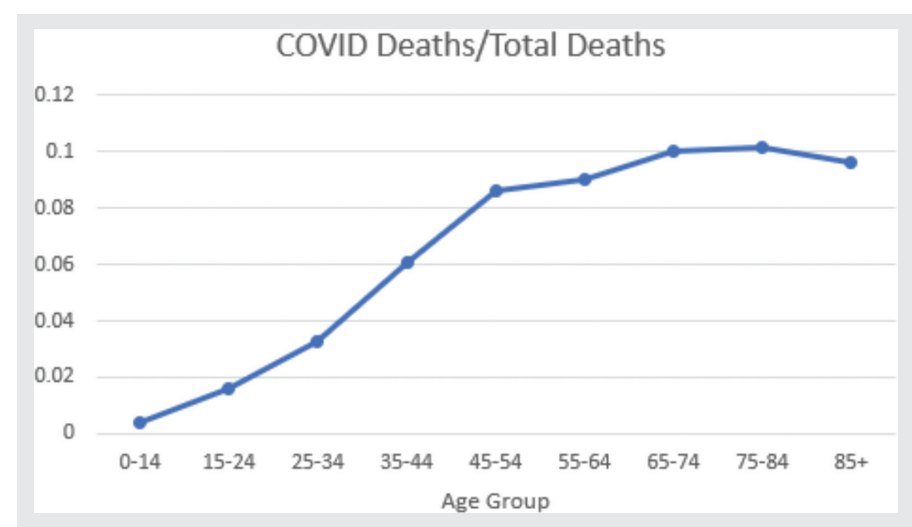

Figure 2. U.S. deaths from COVID as fraction of total deaths from all causes by age group. Data are from CDC. ${ }^{1}$

\section{DIFFICULTIES WITH THE METRIC OF COVID DEATHS}

Figure 2 illustrates deaths attributed to COVID-19 as a fraction of death from all causes stratified by age group. Deaths from all causes is one of the best metrics available. There are rarely disputes as to whether someone died or not. However, the cause of death can be problematic. Cause of death was determined from death certificates. Figure 2 shows that deaths attributed to COVID top out at around $10 \%$ of deaths from all causes even for those aged 85 years and older. This means $90 \%$ or more deaths are attributed to something other than COVID-19. These other causes include heart disease and cancer in the elderly, trauma and drug overdoses in younger age groups, and other infectious disease in all age groups. There is an obvious problem when COVID-19 is present in addition to one or more of these other causes of death. If an 80-yearold presents with an acute myocardial infarction (heart attack) and tests positive for the virus causing COVID19 , is the death from COVID-19 or is the death with COVID-19? This can be a difficult question to answer even if the practitioner filling out the death certificate is motivated by truth; it becomes more problematic when 


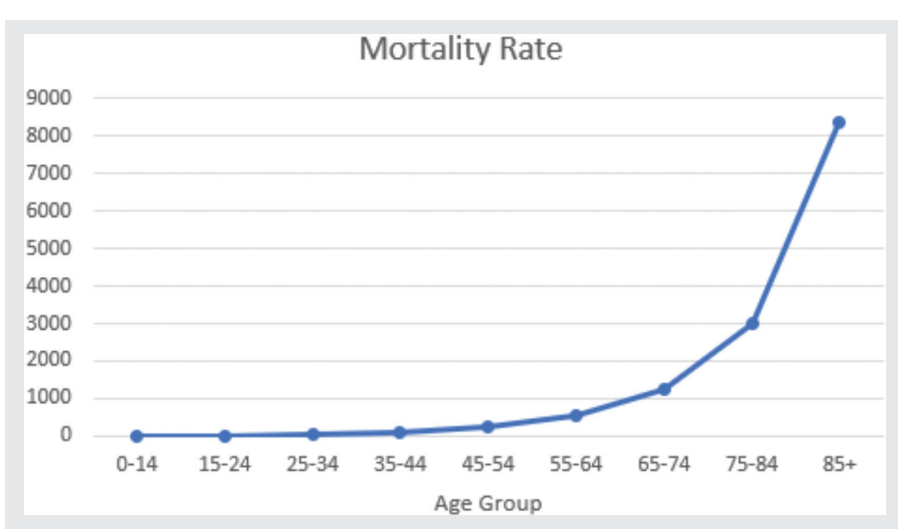

Figure 3. U.S. mortality rates from COVID-19 by age group. Data are from CDC. ${ }^{1}$ Mortality values are deaths per million population since pandemic started.

there are financial or bureaucratic incentives for the practitioner to check the box next to COVID-19.

\section{DifFICULTIES WITH THE METRIC OF COVID CASES}

Figure 3 illustrates U.S. mortality (deaths per million population) attributed to COVID-19 stratified by Age Group. The mortality data show that COVID is more lethal the older one gets. The trend shown in Figure 3 is difficult to reconcile with the plateau in Figure 2 without admitting that the primary cause of death may be a cardiovascular problem (or other leading causes of death) when COVID-19 is present in addition to one of these other leading causes. More important, Figure 3 makes the international obsession over cases impossible to explain. A case of COVID-19 clearly does not have the same implications for 10 -year-old children attending school as for 80-year-old residents of long-term health care institutions including nursing homes.

The definition of a case is problematic. Currently a case is defined as a positive polymerase chain reaction (PCR) test on a nasal swab specimen. PCR tests amplify genetic material by the chain reaction. Each test is specific to a particular sequence of genetic material, but the test has false positives. Dead virus can generate a positive PCR result. Since there is no universally accepted definition of a true positive COVID-19 test, it is unclear how the manufacturers even determine their false positive and false negative rates.

If one had a list of the entire U.S. population, one would have to go through 1 million cases in the age group 0-14 to produce an average expectation of one death. It would only take about 120 names in the age group $85+$ to produce the same result. Policies that treat interactions between K- 6 children the same as interactions between $85+$ residents of nursing homes are clearly not based on scientific data even when the policies are articulated by individuals labeled as scientific experts.

Until everyone has been exposed to the COVID19 virus, mortality rates will necessarily be less than case fatality rates. However, case fatality rates are less useful as a metric than mortality rates, because nobody knows how many cases are asymptomatic, and the group of people who get tested are skewed toward people with more serious disease. Unlike a tape measure, which is a reliable and reproducible metric of distance, the number of cases depends on how many tests are performed and why people choose to be tested. One does not expect the same results from tests on patients with fever as from tests performed as a requirement to attend school. Without information on the age distribution of the people tested, case numbers are useless to predict future deaths. Case numbers are a very unreliable indicator for policy about restrictions on interactions.

Figure 4 is a simplistic view of the transmission of COVID-19. The actual problem is enormously complicated. There are 330 million people in the U.S. give or take. Each person can interact with each other person. The probability of each interaction on any given day is different. Each interaction has a different probability for virus transmission ranging from $0-1$. Figure 4 divides the population into two groups with homogeneous probabilities. The Young and Hale group has a very low case fatality rate. For our purposes, consider it to be zero. The Elderly and Frail group has a significant case fatality rate. For our purposes, consider it to be $15 \%$. There are three types of interactions. The red interactions are between elderly people. The green interactions are between young people. The blue interactions are between one young person and one elderly person. These interactions have much different outcomes. The maximum number of deaths is $15 \%$ of the Elderly population in this scenario. 


\section{Transmission of COVID-19}

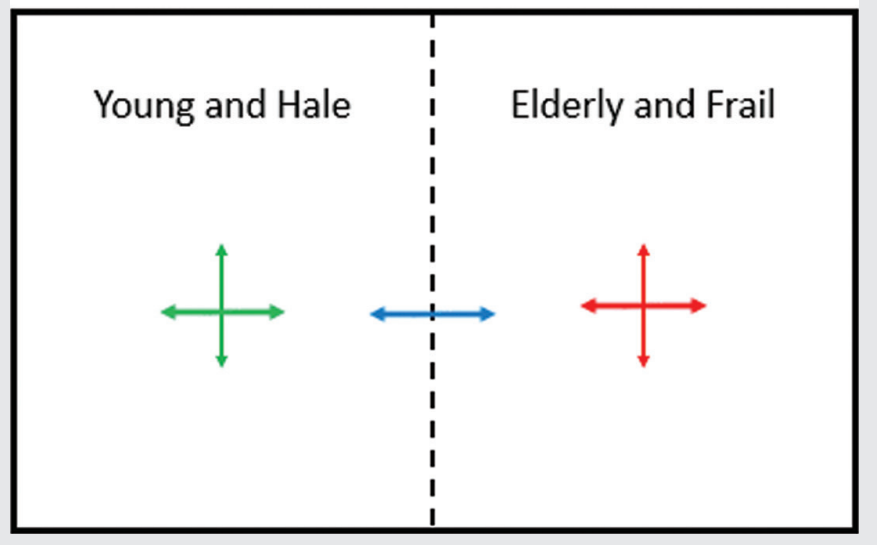

Figure 4. Schematic of COVID-19 transmission in a population divided into a low risk group and a high risk group. See text for details.

This is achieved when all the Elderly population have been exposed. Once this maximum is reached, it no longer matters what happens in the Young population.

\section{RED INTERACTIONS}

This is the most important type of interaction. If this number is not kept below a critical value, then any case entering the Elderly population will expand until all the Elderly population have been exposed and the maximum number of people have died. If this number is kept below the critical value, any entry into the Elderly population through blue interactions will be self-limited. If this number is above the critical value, then the maximum number of people will die irrespective of the numbers of blue and green interactions.

\section{BLUE INTERACTIONS}

If the red interactions are kept below the critical value, then the number of blue interactions determine the number of deaths. The virus will be transmitted from the Young to the Elderly until one of two events occur. If the number of blue interactions is too high compared to the number of green interactions, all the Elderly will be exposed, and the maximum number of people will die. If the number of blue interactions is kept low compared to the number of green interactions, all the Young will be exposed, herd immunity will have been achieved, and the number of deaths will be less than maximum. A low number of green interactions requires a lower number of blue interactions to avoid the maximum number of deaths than is required by a higher number of green interactions. This apparent paradox exists because a low number of green interactions allows blue interactions to occur for a longer duration of time.

\section{GREEN INTERACTIONS}

If the number of blue interactions is high enough for maximal deaths, then lowering the number of green interactions has no effect on total deaths. If the number of blue interactions is low enough to keep the number of deaths below maximum, then lowering the number of green interactions will be counterproductive and increase the number of deaths by increasing the duration of time that virus can spread from the Young to the Elderly prior to achieving herd immunity.

\section{INDISCRIMINATE LOCKDOWNS ARE INEFFECTIVE}

The CDC strategy of containment assumes that the virus can be contained like Ebola. There is no evidence that COVID-19 can be contained. Lockdowns are not sustainable. The virus has resumed its spread following the relaxation of every lockdown no matter how long or strict the lockdown. The virus clearly can survive for long periods of time in a dormant phase. This dormant phase could be in a secondary host species, or in asymptomatic human carriers, or in some kind of biofilm. Lockdowns of Young people (decrease of green interactions) have no benefit and can be counterproductive. Control of interactions among the Elderly (decrease of red interactions) or between the Young and Elderly (decrease of blue interactions) are necessary to reducing the number of deaths. The risks and benefits of social interaction are different for each individual and subjective in nature, so the choices of restricting interactions can be made only by the individuals bearing the risks.

Keywords: COVID-19, mortality rate, cause of death 
Article citation: Berdine G. The Misinterpretation of COVID-19 metrics. The Southwest Respiratory and Critical Care Chronicles 2020;8(36):66-69

From: Department of Internal Medicine, Texas Tech University Health Sciences Center, Lubbock, Texas

Submitted: 9/14/2020

Accepted: 9/22/2020

Conflicts of interest: none

This work is licensed under a Creative Commons

Attribution-ShareAlike 4.0 International License.

\section{REFERENCE}

1. Weekly Updates by Select Demographic and Geographic Characteristics. https://www.cdc.gov/nchs/nvss/vsrr/covid_weekly/ index.htm. 